\title{
¿Cómo se aprende la ortografía de las palabras? Un estudio de comparación de distintas estrategias
}

\author{
How is orthographic knowledge acquired? \\ A comparative study of different strategies
}

\begin{abstract}
Beatriz Diuk
Doctora en Psicología, Universidad Nacional de La Plata, Buenos Aires, Argentina. Investigadora del Consejo Nacional de Investigaciones Científicas y Técnicas. Profesora adjunta de la cátedra Didáctica de la Lengua, carrera de Psicopedagogía, Universidad Nacional de San Martín.
\end{abstract}

\author{
Marina Ferroni \\ Doctora en Psicología, Universidad Nacional de La Plata, Buenos Aires, Argentina. Becaria de Investigación \\ de la Universidad Nacional de San Martín. \\ Jefa de Trabajos Prácticos de la cátedra Didáctica de la Lengua, carrera de Psicopedagogía, \\ Universidad Nacional de San Martín.
}

\section{Milagros Mena}

Licenciada en Psicopedagogía, Universidad Nacional de San Martín, Buenos Aires, Argentina. Profesora Ayudante de la cátedra Introducción a las Prácticas Psicopedagógicas, Carrera de Psicopedagogía, Universidad Nacional de San Martín.

Fecha de recibido: 15/01/2014

Fecha de aceptación: 11/06/2014

\section{Resumen}

El presente trabajo se propuso analizar el impacto de diferentes modalidades de aprendizaje sobre la adquisición de conocimiento de la ortografía de las palabras. Para ello, 30 niños que cursaban el 3.er año de la escolaridad primaria participaron de sesiones de aprendizaje de pseudopalabras con ortografía compleja mediante tres condiciones: lectura, lectura más escritura manuscrita y lectura más escritura con fichas. Tres días después de las sesiones de aprendizaje se evaluaba si efectivamente los niños habían podido almacenar la forma ortográfica de las pseudopalabras a las cuales se vieron expuestos. Para ello se administró una prueba de escritura de las pseudopalabras al dictado y una prueba de decisión léxica en la que los niños debían identificar, entre cuatro opciones, los ítems que se les habían presentado en las sesiones de aprendizaje.

El análisis de los datos mostró que los niños se desempeñaron por encima del nivel de azar en las pruebas de post- test. La comparación de los puntajes en las distintas condiciones reveló diferencias significativas entre las condiciones de lectura y escritura con fichas a favor de esta última. No se encontraron diferencias significativas entre la lectura y la escritura manuscrita ni entre las dos condiciones de escritura.

Los datos obtenidos sugieren que si bien la lectura y la escritura manuscrita constituyen vías para la adquisición de conocimiento ortográfico, la escritura de las palabras con fichas resultó un mecanismo más potente. Se considera que en esta tarea, la búsqueda de cada una de las letras necesarias para formar las pseudopalabras implicaba un uso adicional de recursos atencionales, hecho que impactaría significativamente en el aprendizaje ortográfico.

PALABRAS CLAVE: aprendizaje ortográfico, recodificación fonológica, escritura, procesos motores, recursos atencionales. 


\section{Abstract}

The aim of the present study was to analyze the impact of different learning modalities in the acquisition of orthographic knowledge. Thirty students in the third grade of primary education participated in pseudowords learning sessions, said words having a complex spelling. Such learning sessions were held under three modalities: reading, reading plus handwriting and reading plus spelling with letter tiles. Three days after each learning session, children were post-tested in order to determine whether they had been able to "store" the spelling of those pseudowords they had been exposed to. To such end, children were subjected to a written test where they were requested to spell the words upon dictation as well as to a lexical-decision test where they were requested to identify, out of four options, the pseudowords they had been exposed to during the learning sessions.

Results showed that children performed above chance level in all post-tests. The comparison of scores in the different learning conditions revealed significant differences from the statistic viewpoint between reading and spelling with letter tiles, in favor of the latter. No differences were found either between the reading and the handwriting conditions, or between both handwriting options.

The data thus obtained suggest that even though reading and handwriting give rise to orthographic learning, spelling with letter tiles provides a more powerful learning mechanism. It is considered that as children had to search for each necessary letter in order to spell the pseudowords, this implied the use of additional attentional resources which would be responsible for enhanced orthographic learning.

KEYWORDS: Orthographic learning, phonological recoding, writing, motor processes, attentional resources.

\section{Introducción}

El objetivo del presente trabajo es contribuir a la comprensión de la adquisición de conocimiento ortográfico en español. Más específicamente, el presente estudio se propone analizar el impacto de diferentes modalidades de aprendizaje de palabras con ortografía compleja (lectura, lectura más escritura manuscrita o lectura más escritura con equipo de letras).

Las palabras con ortografía compleja son aquellas palabras que incluyen correspondencias inconsistentes entre fonemas y grafemas, es decir, correspondencias en las cuales a un mismo fonema puede corresponder más de un grafema (en el español rioplatense, por ejemplo, /s/ como $<$ S $>$ o como $<\mathrm{C}>$ ).

Para poder escribir palabras con ortografía compleja de forma convencional, los sujetos deben poseer representaciones ortográficas de esas palabras. Se considera que todo lector experto cuenta con un léxico mental, una especie de diccionario donde alberga las "entradas" correspondientes a cada palabra: estas entradas incluyen representaciones fonológicas (la forma sonora), semánticas (el significado) y ortográficas (la forma escrita convencional) (Perfetti, 1992).

¿Cómo se adquieren estas representaciones ortográficas? Share (1999; 2004; 2008; 2011) ha propuesto la hipótesis de la recodificación fonológica como mecanismo autodidacta, según la cual las representaciones ortográficas de las palabras se construyen a través de las lecturas reiteradas de las mismas. En este marco se considera que existen dos mecanismos fundamentales para leer palabras: las palabras ya conocidas -para las cuales el lector cuenta con representaciones ortográficas- se leen a través de un procesamiento directo sin pasar por una lectura letra por letra; y para las palabras desconocidas o las pseudopalabras -palabras inventadas que no existen en la lengua pero que se pueden leer y que suelen emplearse en situaciones de investigación- se utiliza la recodificación fonológica, es decir, el proceso por el 
cual las letras que componen una palabra son convertidas en sonidos en base a las reglas de correspondencia grafema-fonema, y estos sonidos son ensamblados ("unidos") para su pronunciación (Herrera, Martos \& Defior, 2000; Ferreres, Martínez Cuitiño, Jacubovich, Olmedo \& López, 2003; Signorini \& Piacente, 2001).

La propuesta de Share (1999; 2004; 2008; 2011), entonces, consiste en considerar que las palabras pasan por el siguiente proceso: cuando son no familiares para el sujeto, éste las recodifica fonológicamente. Luego de sucesivas remodificaciones, los sujetos forman representaciones ortográficas de esas palabras y las mismas se convierten en palabras familiares que pueden leerse por la vía directa.

Para poner a prueba la hipótesis de la recodificación fonológica como mecanismo autodidacta, Share (1999) diseñó una situación experimental en la cual niños en proceso de adquisición de la lengua escrita leen en voz alta textos breves que contienen una pseudopalabra. Dado que una pseudopalabra es una palabra inventada, necesariamente su forma ortográfica es nueva para el niño. El objetivo del experimento es ver si el hecho de leerlas dentro de los textos permite que se formen representaciones ortográficas. Para ello, tres días después de la situación de lectura, se evaluaba si efectivamente los niños habían aprendido la forma ortográfica de la pseudopalabra que se les había presentado. Para ello readministraban dos pruebas: una prueba de escritura de la pseudopalabra al dictado y una prueba de decisión léxica; una prueba en la que se presenta a los niños diversas pseudopalabras y deben identificar la que habían leído dentro del texto.

Los resultados de diversos estudios que utilizaron esta situación experimental mostraron que los sujetos habían almacenado las representaciones ortográficas de las pseudopalabras que habían recodificado, ya que el desempeño en las pruebas de decisión léxica y de escritura del post-test estuvo por encima del nivel de azar.

La hipótesis propuesta por Share $(1995 ; 1999 ; 2004 ; 2008 ; 2011)$ ha sido testeada en ortografías transparentes, en las cuales las correspondencias entre fonemas y grafemas poseen un alto grado de consistencia (Share, 1999; 2004; Share \& Shalev, 2004) y en ortografías opacas en las cuales existe un alto número de palabras irregulares que no se ajustan a las reglas de correspondencia grafema-fonema (Bowey \& Miller, 2007; Bowey \& Muller, 2005; Cunningham, 2006; Cunningham, Perry, Stanovich \& Share, 2002; Kyte \& Johnson, 2006; Nation, Angell \& Castles, 2007). En todas estas investigaciones se obtuvieron resultados que otorgan sustento empírico a la hipótesis de la recodificación fonológica como mecanismo autodidacta.

Ahora bien, los resultados de algunos estudios que utilizaron la situación experimental de Share (Nation et al., 2007; Ferroni \& Diuk, en prensa) sugirieron que no puede sostenerse una "versión fuerte" de la hipótesis de la recodificación fonológica como mecanismo autodidacta: se encontró que los sujetos forman representaciones ortográficas de algunas pseudopalabras pero no de otras.

Así, por ejemplo, en el estudio de Ferroni y Diuk (en prensa), se pidió a 46 niños que cursaban el 3.er grado de la educación primaria que leyeran 6 narraciones breves dentro de las cuales se presentaban pseudopalabras con ortografía compleja. Cuando se promedió el desempeño en el post-test de todas las pseudopalabras, se obtuvo evidencia a favor de la hipótesis de la recodificación fonológica como mecanismo de autoaprendizaje. Sin embargo, un análisis detallado de los resultados obtenidos en cada una de las pseudopalabras mostró que el desempeño se encontraba afectado por las características de las correspondencias entre los sonidos y las letras que se debían representar. Más específicamente, se vio que cuando entre los posibles grafemas a representar alguno era mucho más frecuente que los otros (por ejemplo, para el fonema /s/, el grafema $<\mathrm{S}>$ es mucho más frecuente que $<\mathrm{C}>0<\mathrm{Z}>$ ), los niños no almacenaban las pseudopalabras con los grafemas menos frecuentes $(<\mathrm{C}>0<\mathrm{Z}>$ ). 
En la prueba de dictado posterior a la lectura, los sujetos tendían a escribir la pseudopalabra con la letra más frecuente (por ejemplo, escribían Tusial) a pesar de haberla leído con una letra poco frecuente (Tuzial). Esto no sucedía cuando los dos grafemas posibles tenían una frecuencia similar (por ej., para el sonido /II/ los grafemas $\langle\mathrm{Y}\rangle \mathrm{y}\langle\mathrm{LL}\rangle$ tienen una frecuencia muy parecida). En estos casos, los niños tendían a almacenar la pseudopalabra con el grafema que habían leído (tanto los niños que habían leído Yutosa como Llutosa podían almacenar la pseudopalabra de manera correcta).

Para interpretar estos resultados se consideró que un grafema mucho más frecuente que los posibles grafemas alternativos dificulta el recuerdo de los menos frecuentes, al menos en la situación experimental propuesta por Share (1999) en la cual los niños se limitaban a leer las pseudopalabras.

¿Cómo se adquieren entonces las representaciones ortográficas de las palabras con grafemas poco frecuentes? Existe un considerable número de estudios que exploraron otras modalidades de aprendizaje de la forma ortográfica de las palabras, más allá de la lectura. Algunos de estos trabajos proponen que sería la escritura un mecanismo con mayor potencia.

Así por ejemplo, Shahar-Yames y Share (2008) realizaron un estudio con niños de 3.er grado hablantes de hebreo, a quienes se les presentaron pseudopalabras con ortografía arbitraria mediante tres condiciones: escritura, lectura y una condición control. Los sujetos de la condición de escritura mostraron un mayor aprendizaje de la forma ortográfica de las palabras que los demás.

Se ha sostenido que la escritura de las palabras produce un impacto en el aprendizaje ortográfico dada la importancia que la programación motora propia de esta tarea tendría en la formación de representaciones ortográficas de las palabras (Kurlat \& Politis, 2005). Se sugiere que los movimientos realizados al trazar las letras de las palabras generarían patrones motores que conectarían las representaciones fonológicas (las representaciones de los sonidos de las palabras) y las nuevas representaciones ortográficas (Bara, Gentaz \& Colé; 2007; Bara, Gentaz, Colé \& Sprenger-Charolles, 2004; Berninger et al., 1992; Cunningham \& Stanovich, 1991; Fredembach, de Boisferon \& Gentaz, 2009; Shahar-Yames \& Share, 2008; Share, 2011).

Ahora bien, se han formulado otras hipótesis para explicar cómo la escritura contribuiría a formar representaciones ortográficas. Se ha sostenido que el aspecto fundamental puede no ser la programación motora que se da en la escritura manuscrita sino el nivel de atención que demanda la escritura, dado que es más lenta y secuenciada que la lectura. Esta mayor atención a la tarea daría lugar a mayores posibilidades de formar y almacenar representaciones ortográficas de las palabras. Según esta hipótesis, la escritura manuscrita tendría el mismo efecto que escribir palabras utilizando equipos de letras.

Vaughn, Schumm y Gordon (1992; 1993) y Masterson y Apel (2006) en estudios realizados con sujetos de $2 .^{\circ}$ grado y de preescolar respectivamente encontraron que el aprendizaje ortográfico por medio de la escritura de palabras era independiente de la modalidad de producción (escritura manuscrita o en un teclado de computadora).

Por su parte, Berninger y colaboradores (1998) llevaron a cabo sesiones de entrenamiento ortográfico en las cuales los niños debían escribir palabras con diferentes tipos de complejidad ortográfica en el teclado de una computadora o a mano. Los investigadores observaron que, si bien las palabras simples (es decir, con correspondencias sencillas entre fonemas y grafemas) fueron mejor aprendidas mediante la escritura manual, las palabras con ortografía compleja fueron mejor aprendidas en la condición de escritura en un teclado.

A pesar de que existe un considerable número de estudios que han analizado diferentes formas de aprender la ortografía de las palabras, no se conocen en español estudios que hayan comparado la adquisición de representaciones ortográficas a través de distintas situaciones de aprendizaje. Por lo tanto, el presente estudio se propone comparar la eficacia, sobre la 
adquisición de representaciones ortográficas, de tres condiciones: exclusivamente la lectura, la lectura más la escritura manuscrita, o la lectura más la escritura con un equipo de letras. Con esto se busca lo siguiente:

1) Analizar si la escritura, además de la lectura, da lugar a la formación y el almacenamiento de representaciones ortográficas de las palabras.

2) Explorar la relación entre la escritura y la formación de representaciones ortográficas comparando una situación de aprendizaje ortográfico mediante la escritura manuscrita, la cual implica la posible reproducción de patrones grafo-motores, con otra situación (escritura con fichas) en la cual se estaría involucrando el procesamiento secuencial de alta demanda atencional propio de la escritura pero sin el componente de un patrón grafo-motor específico.

\section{Metodología}

\section{Sujetos}

Este estudio se llevó a cabo con 32 sujetos que cursaban el 3.er grado de enseñanza primaria en una escuela de Buenos Aires. Con anterioridad al comienzo de las sesiones de evaluación, las familias de los niños firmaron un consentimiento informado expresando su conformidad para que éstos participaran en el proyecto de investigación. Los niños participantes manifestaron su asentimiento en forma verbal y en todo momento la evaluadora estuvo atenta a que no se produjera ningún tipo de malestar que justificara la exclusión de algún niño de la evaluación. Dos niños no pudieron completar las tres sesiones de aprendizaje y por lo tanto fueron excluidos de la muestra. La misma quedó conformada definitivamente por 30 sujetos. Todos los participantes eran hispanohablantes nativos.

Mediante la administración de una prueba de lectura de pseudopalabras (Test LEE, Defior Citoler et al., 2006) se controló que los sujetos tuvieran un nivel de recodificación fonológica acorde a su edad. Se observó un desempeño superior al 91\% (DE =6,27), hecho que sugiere un adecuado desarrollo de los mecanismos de recodificación fonológica de los sujetos de la muestra escogida.

\section{Diseño}

Se trata de un diseño intrasujetos en el cual los niños participaban de sesiones de aprendizaje de pseudopalabras con ortografía compleja en tres diferentes condiciones: lectura, lectura más escritura manuscrita y lectura más escritura con equipos de letras.

Cada participante fue expuesto a 4 pseudopalabras en cada una de las tres condiciones (total de 12 palabras). Tanto las pseudopalabras meta (es decir, las pseudopalabras seleccionadas para que los niños aprendieran en las sesiones de aprendizaje) como cada una de las condiciones de aprendizaje iban rotando entre sesiones para neutralizar cualquier variable sin controlar. Asimismo, cada pseudopalabra meta aparecía en una de sus dos versiones homófonas (por ej. Gientado o Jientado). La mitad de la muestra era expuesta a una versión de la pseudopalabra y la otra mitad a la otra.

Las pseudopalabras meta se encontraban incluidas en oraciones, por ejemplo, Silfosa era una nube que no podía viajar por el cielo.

Cada sujeto participó de 3 sesiones de aprendizaje ortográfico. Tres días después de realizadas las sesiones se evaluaba si efectivamente los sujetos habían aprendido las pseudopalabras meta. 


\section{Sesiones de aprendizaje}

Lectura. Los niños leían en voz alta un par de oraciones en las cuales se encontraban incluidas las pseudopalabras meta (por ejemplo: Hecna era una bruja. Los chicos le tenían miedo a Hecna). Los sujetos leían en voz alta el par de oraciones 2 veces (total de 4 veces que recodificaban la pseudopalabra meta).

Lectura y escritura manuscrita. Los niños leían una oración que incluía la pseudopalabra meta (por ejemplo: Tucial es el malabarista del circo más grande del mundo) y luego debían escribir la pseudopalabra de forma manuscrita 2 veces a partir del recuerdo. En caso de error en la escritura, la evaluadora realizaba una "devolución" (feedback) al sujeto garantizando una recodificación precisa.

Lectura y escritura con fichas. En esta condición, los niños leían una oración que incluía la pseudopalabra meta (por ejemplo: Pibauno es un palomo que le roba comida a sus amigos) y luego debían formar dicha pseudopalabra con fichas que contenían letras. Una vez que formaban la pseudopalabra, la evaluadora volvía a mezclar todas las letras (incluidas las que se habían utilizado segundos antes para el armado de la pseudopalabra meta la primera vez) y el niño debía realizar una nueva búsqueda para formar la pseudopalabra por segunda vez. En caso de error, la evaluadora realizaba una devolución al sujeto garantizando una recodificación precisa.

\section{Pseudopalabras meta}

Para la selección de las pseudopalabras que los niños debían aprender en las sesiones de aprendizaje se realizó un estudio previo en el cual a 27 sujetos que cursaban $2 . .^{\circ}$ grado se les presentaron pares de pseudopalabras con igual sonido pero diferente escritura (por ejemplo: Alorajis y Aloragis). Este par de pseudopalabras siempre incluía una correspondencia inconsistente, es decir, un sonido que puede ser representado por más de una letra. Luego de presentadas las pseudopalabras, se preguntaba a los sujetos qué pseudopalabra se parecía más a una palabra real del idioma español. El objetivo de este estudio fue elegir para las sesiones de aprendizaje pseudopalabras que no fueran muy diferentes a los patrones fonológicos y ortográficos de la lengua, hecho que haría muy llamativa a la pseudopalabra y, por ende, más fácilmente reconocible; ni tampoco muy parecida a alguna palabra del idioma, hecho que haría a las pseudopalabras meta fácilmente asociables a alguna palabra existente. Se registraron las respuestas de los niños y se seleccionaron 12 pares de pseudopalabras que se convirtieron en las palabras meta, es decir, pseudopalabras que se incluirían en las sesiones para que los sujetos aprendieran.

\section{Post-test}

Tres días después de las sesiones de aprendizaje de las pseudopalabras meta se administraron dos pruebas (decisión léxica y dictado de las pseudopalabras meta) para medir si se había producido aprendizaje ortográfico y para analizar si se habían producido diferencias entre las distintas condiciones de aprendizaje.

\section{Decisión léxica}

Se presentó a cada sujeto una serie de láminas en las cuales se encontraban la pseudopalabra meta (por ejemplo, Silfosa), su pseudohomófono (Cilfosa) y dos distractores (Gilfosa y Filsosa). Los dos distractores fueron diseñados con diferentes criterios: uno de ellos contenía una letra gráficamente similar al grafema meta (Gilfosa cuando la pseudopalabra meta era Cilfosa) y el otro contenía las mismas letras que la pseudopalabra meta pero dos de ellas se encontraban rotadas (Filsosa cuando la pseudopalabra meta era Silfosa). Ambos constituían distractores visuales. 
Para la mitad de la muestra, una versión de la pseudopalabra era la "correcta"; en tanto para la otra mitad de la muestra, el objetivo "correcto" era la escritura alternativa de la pseudopalabra. Los ítems fueron presentados en una fuente diferente a la utilizada en los textos originales para que el reconocimiento no se viera facilitado por las similitudes en la tipografía.

\section{Dictado}

En esta prueba, la evaluadora preguntaba a los sujetos si recordaba alguno de los nombres de los personajes que había leído en las oraciones (pseudopalabra meta). Si el niño o niña no recordaba la pseudopalabra meta la evaluadora le facilitaba la primera sílaba de la misma. Si, aun así el sujeto no recordaba la pseudopalabra meta, la evaluadora la decía y el niño debía escribirla. Se recordaba a los sujetos que debían tratar de escribir la palabra respetando la forma que había leído. Se asignaba un punto a cada pseudopalabra meta escrita de la misma manera que había aparecido en las oraciones.

\section{Procedimiento}

La primera autora de este trabajo fue la evaluadora de los sujetos de la muestra.

Cada sujeto participó de 4 sesiones consecutivas separadas entre sí por intervalos de tres días. En la primera sesión, cada niño participó de una sesión de aprendizaje de 4 pseudopalabras en una de las 3 condiciones planteadas. En la segunda y tercera sesión, se realizó el post-test de las pseudopalabras de la sesión anterior más la exposición a 4 nuevas pseudopalabras en otra de las condiciones experimentales planteadas. En la sesión 4 se administró el post-test de la sesión 3.

Como se indicó más arriba, las sesiones se llevaron a cabo respetando un intervalo de tres días. Las mismas tuvieron lugar en horario escolar, en un aula vacía de la escuela a la cual asistían los niños.

\section{Resultados}

En primer lugar, se analizaron las distribuciones de las puntuaciones obtenidas en las tareas administradas. Este análisis permitió observar que las medidas que se alejaron significativamente de la distribución normal asintótica fueron los resultados de las pruebas de dictado en la condición de lectura más escritura a mano $(Z$ de Kolmogorov-Smirnov $=1.370 ; p=$ .047) y en la condición de lectura más escritura con fichas ( $Z$ de Kolmogorov-Smirnov = 1.549; $p=.016$ para la prueba de decisión léxica). Los análisis de datos que incluían las medidas señaladas se realizaron, consecuentemente, mediante pruebas estadísticas no paramétricas.

Para el análisis de los resultados obtenidos en el post-test se consideró que puntajes superiores al $50 \%$ en la prueba de dictado de las pseudopalabras meta y puntajes superiores al $25 \%$ en la prueba de decisión léxica corresponden a un desempeño por encima del nivel de azar. Esto responde al hecho de que en la prueba de dictado los sujetos pueden optar por dos formas posibles de escribir el ítem en tanto en la tarea de decisión léxica hay cuatro posibles respuestas. En la Tabla 1 se presentan los datos obtenidos en el post-test (medias y desvíos estándar en porcentajes). 


\section{Tabla 1}

Estadísticos descriptivos de las medidas obtenidas en el post-test de aprendizaje ortográfico (decisión léxica y dictado)

\begin{tabular}{lcccc}
\hline \multicolumn{1}{c}{ Condición } & \multicolumn{2}{c}{$\begin{array}{c}\text { Decisión léxica } \\
M(D E)\end{array}$} & \multicolumn{2}{c}{$\begin{array}{c}\text { Dictado } \\
\boldsymbol{M}(\boldsymbol{D} \text { (DE) }\end{array}$} \\
\hline Lectura & 52.50 & 31.72 & 70.00 & 26.58 \\
Lectura y escritura manuscrita & 47.50 & 30.33 & 64.65 & 30.26 \\
Lectura y escritura con fichas & 60.41 & 32.90 & 82.60 & 20.55 \\
\hline
\end{tabular}

Con el objeto de comparar la eficacia de las distintas modalidades de aprendizaje en el posterior desempeño de los niños, se realizó una serie de pruebas t de muestras relacionadas comparando las medias obtenidas en la prueba de decisión léxica y una serie de pruebas no-paramétricas de contraste ( $U$ de Wilcoxon) entre las medias obtenidas en las pruebas de dictado. Los resultados mostraron diferencias significativas entre los resultados de la condición de lectura y la condición de escritura con fichas a favor de esta última condición $(Z=2.101$. p= .036 para el desempeño en la prueba de escritura del post-test y t $(22)=2.235$. $p=.029$ para el desempeño en la prueba de decisión léxica del post-test). No se encontraron diferencias significativas entre la condición de escritura a mano y lectura $(Z=.982 p=.022)$ ni entre las condiciones de escritura manuscrita y escritura con fichas $(Z=1.712 . p=.086)$.

\section{Discusión}

El objetivo del presente trabajo fue contribuir a la comprensión de la adquisición de conocimiento ortográfico en español. Se buscó, específicamente, analizar el impacto de diferentes modalidades de aprendizaje (lectura, lectura más escritura o lectura más escritura con equipo de letras) sobre el aprendizaje de pseudopalabras con ortografía compleja.

Para ello, 30 sujetos hispanohablantes que cursaban el 3.er grado de la escuela primaria participaron de sesiones de aprendizaje ortográfico mediante tres condiciones diferentes. Tres días después de las sesiones de aprendizaje, los sujetos se sometían a una prueba de decisión léxica y a una prueba de dictado de las pseudopalabras presentadas.

Los resultados mostraron, en primer lugar, que los sujetos se desempeñaron por encima del nivel del azar en el post-test en las tres condiciones (lectura, lectura escritura manuscrita y lectura y escritura con equipo de letras). Esto significa que en las tres condiciones hubo algún nivel de aprendizaje de la forma ortográfica de las pseudopalabras presentadas.

Los datos obtenidos en la condición de lectura, al igual que en estudios anteriores, proporcionan evidencia empírica sobre la hipótesis de la recodificación fonológica como mecanismo autodidacta (Bowey \& Muller, 2005; Cunningham, 2006; Cunningham, Perry, Stanovich, \& Share, 2002; Kyte \& Johnson, 2006; Nation, Angell \& Castles, 2007; Share, 1999; 2004; Share \& Shalev, 2004).

Por otro lado, los resultados del presente estudio sugieren que existen otras formas además de la lectura a partir de las cuales los sujetos forman representaciones ortográficas de las palabras a las que se ven expuestos. En efecto, los niños también evidenciaron haber aprendido la forma ortográfica de las pseudopalabras que habían escrito a mano o con equipos de letras. 
Ahora bien, los datos obtenidos no mostraron diferencias significativas entre aquellas pseudopalabras que los sujetos habían leído solamente y aquellas pseudopalabras que habían escrito a mano. Es decir, contrariamente a otras investigaciones realizadas (Cunningham, 1990; Yahar Shames \& Share, 2004), no se encontró evidencia de que la escritura manuscrita fuera una forma de aprendizaje con mayor potencia que la lectura.

Los estudios en los que se halló que la escritura manuscrita daba lugar a un mayor aprendizaje que la lectura interpretaron este resultado de dos maneras.

Por una parte, los autores explicaron los resultados como consecuencia de la incidencia de los procesos motores en el aprendizaje ortográfico (Cunningham, 1990; Yahar Shames y Share, 2004). Los resultados obtenidos en el presente estudio no otorgan apoyo a esta hipótesis. Por otra parte, no resultaría erróneo entonces plantear una explicación alternativa al mayor aprendizaje encontrado en estudios previos en la situación de escritura por sobre la de lectura. Esta explicación se relaciona con el hecho de que entre los niños pequeños, para quienes la escritura no constituye un mecanismo automático, la condición de aprendizaje de pseudopalabras mediante la escritura daría lugar a que los sujetos realizaran un análisis más exhaustivo y atento de la palabra, lo que permitiría destinar mayores recursos atencionales en comparación al análisis "abreviado" que tendría lugar durante la lectura (Yahar Shames \& Share, 2004).

Ahora bien, los sujetos que participaron del presente estudio reproducían muy velozmente la forma escrita de las pseudopalabras. Este hecho permitiría pensar que la automatización del proceso de escritura manuscrita que estos niños pusieron en evidencia determinó que no se destinaran mayores recursos atencionales al escribir que al leer.

En este sentido, los datos obtenidos sí señalaron la existencia de diferencias estadísticamente significativas entre la condición de lectura y la condición de escritura con equipo de letras. Sería posible pensar que dicha modalidad permite que los sujetos analicen detenidamente y de manera exhaustiva las pseudopalabras a aprender. La condición de escritura con equipo de letras, en la cual los niños debían armar las pseudopalabras buscando cada letra originó que tuvieran que detenerse con mayor atención en la construcción de las pseudopalabras.

Esta idea coincide con el planteo de Berninger y colaboradores (1998) de que el aprendizaje ortográfico podría depender fuertemente de los recursos de atención de un sujeto.

El presente trabajo posee importantes implicancias pedagógicas. En primer lugar, los resultados arrojados en el presente estudio sugieren que existen formas diversas de aprender la ortografía de las palabras.

En segundo lugar, los datos del presente trabajo proporcionan evidencia de que las modalidades de aprendizaje ortográfico con mayor potencia no serían aquellas con un alto nivel de automatización, como la lectura y la escritura, sino aquellas modalidades en las cuales sea necesario realizar un análisis exhaustivo y pausado de los grafemas que incluyen las palabras a aprender. 


\section{Bibliografía}

Bara, F., Gentaz, E. \& Colé, P. (2007). Haptics in learning to read with children from low socioeconomic status families. British Journal of Developmental Psychology, 25(4), 643-663.

Bara, F., Gentaz, E., Sprenger-Charolles \& Colé, P. (2004). The visuo-haptic and haptic exploration of letters increases the kindergarten-children's reading acquisition. Cognitive Development, 19, 433-449.

Berninger, V., Vaughan, K., Abbott, R., Brooks, A., Abbott, S., Reed, E., Rogan, L. \& Graham, S. (1998). Early intervention for spelling problems: Teaching functional spelling units of varying size with a multiple-connections framework. Journal of Educational Psychology, 90, 587-605.

Berninger, V., Yates, C., Cartwright, A., Rutberg, J., Remy, E. \& Abbott, R. (1992). Lower-level developmental skills in beginning writing. Reading and Writing. An Interdisciplinary Journal, 4, 257-280.

Bowey, J. A. \& Miller, R. (2007). Correlates of orthographic learning in third-grade children's silent reading. Journal of Research in Reading, 30, 115-128.

Bowey, J. A. \& Muller, D. (2005). Phonological recoding and rapid orthographic learning in thirdgraders silent reading: A critical test of the self-teaching hypothesis. Journal of Experimental Child Psychology, 92, 203-219.

Cunningham, A. E. (2006). Accounting for children's orthographic learning while reading text: Do children self-teach? Journal of Experimental Child Psychology, 95, 56-77.

Cunningham, A. E. \& Stanovich, K. E. (1990). Early spelling acquisition: Writing beats the computer. Journal of Educational Psychology, 82, 159-162.

Cunningham, A. E., Perry, K. E., Stanovich, K. E. \& Share, D. L. (2002). Orthographic learning during reading: Examining the role of self-teaching. Journal of Experimental Child Psychology, 82, 185-199.

Defior Citoler, S., Fonseca, L., Gottheil, B., Aldrey, A., Jiménez Fernández, G., Pujals, M., Rosa, G. \& Serrano Chica, F. D. (2006). Test de lectura y escritura en español. Buenos Aires, Paidós.

Ferreres, A., Martínez Cuitiño, M., Jacubovich, S., Olmedo, A. \& López, C. (2003). Las alexias y los modelos de doble ruta de lectura en hispanohablantes. Revista Argentina de Neuropsicología, 1, 37-52.

Ferroni, M. \& Diuk, G. (en prensa). Recodificación fonológica y formación de representaciones ortográficas en español. Revista Psykhé.

Fredembach, B., de Boisferon, A. H. \& Gentaz, E. (2009). Learning of arbitrary association between visual and auditory novel stimuli in adults: The "bond effect" of haptic exploration. PLoS ONE, 4(3), e4844. Obtenido de www.plosone.org el 6 de mayo de 2010.

Herrera, L., Martos, F. \& Defior, S. A. (2000). Influencia de las características del sistema ortográfico español en el aprendizaje de la escritura de palabras. Estudios de Psicología, 67, 55-63.

Kurlat, V. \& Politis, D. (2005). Alteraciones de la programación motora de la escritura. Revista española de Neuropsicología, 7(2), 100-115.

Kyte, C. S. \& Johnson, C.J. (2006). The role of phonological recoding in orthographic learning. Journal of Experimental Child Psychology, 93, 166-185.

Masterson, J. \& Apel, K. (2006). Effect of Modality on Spelling Words Varying in Linguistic Demands. Developmental Neuropsychology, 29(1), 261-277. 
Nation, K., Angell, P. \& Castles, A. (2007). Orthographic learning via self-teaching in children learning to read English: Effects of exposure, durability, and context. Journal of Experimental Child Psychology, 96, 71-78.

Perfetti, C. A. (1992). The representation problem in reading acquisition. En P. B. Gough, L. C. Ehri y R. Treiman (Eds.), Reading acquisition. Hillsdale, NJ: Erlbaum.

Shahar-Yames, D. \& Share, D. L. (2008). Spelling as a self-teaching mechanism in orthographic learning. Journal of Research in Reading, 31, 22-39.

Share, D. L. (1995). Phonological recoding and self-teaching: sine qua non of reading acquisition. Cognition, 55, 151-218.

Share, D. L. (1999). Phonological recoding and orthographic learning: A direct test of the selfteaching hypothesis. Journal of Experimental Child Psychology, 72, 95-129.

Share, D. L. (2004). Orthographic learning at a glance: On the time course and developmental onset of self-teaching. Journal of Experimental Child Psychology, 87, 267-289.

Share, D. L (2008). Orthographic learning, phonology and the self-teaching hypothesis. Advances in Child Development and Behavior, 36, 31-82.

Share, D. L. (2011). On the role of phonology in reading acquisition: The self teaching hypothesis. En S. A. Brady, D. Braze \& C. A. Fowler (Eds.), Explaining individual differences in reading: Theory and evidence. (pp. 45-68). New York, NY: Psychology Press.

Share, D. L. \& Shalev, C. (2004). Self-teaching in normal and disabled readers. Reading and Writing, 17(7-8), 769-800.

Signorini, A. \& Piacente, T. (2001). Adquisición de la lectura en español: Las habilidades de procesamiento de palabras en lectores iniciales. Revista Irice, 15, 5-29.

Vaughn, S., Schumm, J. \& Gordon, J. (1992). Early spelling acquisition: Does writing really beat the computer? Learning Disability Quarterly, 15, 223-228.

Vaughn, S., Schumm, J. \& Gordon, J. (1993). Which motoric condition in most effective for teaching spelling to students with and without learning disabilities? Journal of Learning Disabilities, 26(3), 191-198. 